\title{
Anatomical analysis of the distribution patterns of occipital cutaneous nerves and the clinical implications for pain management
}

This article was published in the following Dove Press journal: Journal of Pain Research

\author{
Hyun-Jin Kwon ${ }^{1,2}$ \\ Hong-San $\mathrm{Kim}^{3}$ \\ Jehoon $\mathrm{O}^{2}$ \\ Hyo Jong Kang ${ }^{4}$ \\ ji Yeon Won ${ }^{4}$ \\ Hun-Mu Yang ${ }^{2}$ \\ Shin Hyung Kim ${ }^{4}$ \\ You-Jin $\mathrm{Choi}^{2}$ \\ 'Division in Anatomy and \\ Developmental Biology, Department \\ of Oral Biology, Yonsei University \\ College of Dentistry, Seoul, Republic \\ of Korea; ${ }^{2}$ Department of Anatomy, \\ Yonsei University College of Medicine, \\ Seoul, Republic of Korea; ${ }^{3}$ Department \\ of Anatomy, School of Medicine, Ewha \\ Womans University, Seoul, Republic of \\ Korea; ${ }^{4}$ Department of Anesthesiology \\ and Pain Medicine, Anesthesia and \\ Pain Research Institute, Yonsei \\ University College of Medicine, Seoul, \\ Republic of Korea
}

Correspondence: You-jin Choi Department of Anatomy, Yonsei University College of Medicine, 50-I Yonsei-ro, Seodaemun-gu, Seoul 03722,

Republic of Korea

$\mathrm{Tel}+82222281649$

Fax +82 23650700

Email CYJ7797@yuhs.ac

\begin{abstract}
Purpose: Establishing the distribution patterns of occipital cutaneous nerves may help us understand their contribution to various occipital pain patterns and ensure that a proper local injection method for treatment is employed. The aim of this study was to demonstrate the detailed distribution patterns of the greater occipital nerve (GON), lesser occipital nerve (LON), and third occipital nerve (TON) using the modified Sihler's staining technique.
\end{abstract}

Methods: Ten human cadavers were manually dissected to determine the nerve distributions. Specimens from eight human cadavers were treated using the modified Sihler's staining.

Results: In all cases, distinct GON branches proceeded laterally and were intensively distributed in the superolateral area from their emerging point. Very thin twigs were observed at the middle-trisected area, which had a fan-like shape, in the middle-upper occipital region.

Conclusion: The LON and TON distribution areas were biased to the lateral side below the superior nuchal line, although these nerves exhibited multiple interconnections or overlapping areas with the GON. Furthermore, a nerve rarified zone in the shape of an inverted triangle was identified in the middle occipital area. Our findings improve our understanding of the occipital nerve anatomy and will aid in the management of occipital pain in clinical practice.

Keywords: greater occipital nerve, lesser occipital nerve, third occipital nerve, Sihler's stain, whole mount nerve staining, occipital neuralgia

\section{Introduction}

The greater occipital nerve (GON) arises from fibers of the dorsal primary ramus of the second cervical nerve and, to a lesser extent, from fibers of the third cervical nerve. ${ }^{1}$ The GON provides cutaneous innervation to most of the posterior scalp. ${ }^{1} \mathrm{~A}$ GON block is commonly performed to provide anesthesia and analgesia to the occipital area., Especially, a positive response to a GON block is necessary to establish a diagnosis of occipital neuralgia. ${ }^{3-5}$ In the modern ultrasound era, GON blocks performed under ultrasound guidance have gained popularity in clinical practice. ${ }^{6,7}$ However, GON blocks based on surface anatomical landmarks are still used frequently, since such blocks can be performed easily and show a relatively high success rate. ${ }^{6}$ There are variable landmark methods for GON block that have been proposed over the last few decades based on anatomical studies. ${ }^{1-7}$ Making an injection just medial to the palpated occipital artery at the level of the superior nuchal line (SNL) or making an injection at one-quarter of the distance along a line connecting the external occipital protuberance to the mastoid (or $2 \mathrm{~cm}$ lateral and $2 \mathrm{~cm}$ inferior to the external occipital protuberance) are commonly used clinical practices. Some authors have described this point as being 
$1.5 \mathrm{~cm}$ lateral and $2-2.5 \mathrm{~cm}$ inferior to the external occipital protuberance. ${ }^{3}$ However, because great individual variability in the presentation of the GON has been described in literature, some physicians prefer to use an infiltration method in a fan-like manner for GON block. ${ }^{3,6,7}$

Although several gross anatomical studies have suggested the location of the occipital nerves, detailed information regarding the final distributions of these nerves, including the locations of intensively distributed areas in the entire posterior scalp area remain unclear. This might be attributed to limitations of the manual dissection method and to clinical physicians' concentrated interest in the injection points at the proximal site. Therefore, studies examining the detailed distributions of the occipital nerves are necessary, as they may help explain the roles these nerves play in various pain patterns that originate from the upper cervical segments, as well as aid in the establishment of optimal treatment strategies including the best injection method.

One approach that can be employed to study nerve distributions is Sihler's staining. Sihler's staining is a whole-mount nerve staining technique that renders soft tissue translucent or transparent while staining the peripheral nerve axons. ${ }^{8,9}$ Using this method, the entire nerve supply patterns within the skeletal muscles, mucosa, and subcutaneous layer can be mapped. ${ }^{8,9}$ The unique advantage of Sihler's staining over other anatomical methods is that all the nerves within the stained specimen can be visualized without displacing any of the small nerve twigs. ${ }^{8-11}$

The purpose of this anatomical study was to demonstrate the detailed distribution patterns of major occipital cutaneous nerves, including the GON, lesser occipital nerve (LON), and third occipital nerve (TON), by performing modified Sihler's staining of whole occipital specimens harvested from fixed cadavers, with the aim of providing clinically relevant information for pain management.

\section{Methods}

This cadaveric anatomical study was approved by the institutional review board of Severance Hospital, Yonsei University Health System (No 4-2017-1180). All 18 cadavers utilized in the present study were legally donated to the Surgical Anatomy Education Center at Yonsei University College of Medicine.

\section{Specimen harvesting and Sihler's staining}

Ten fixed cadavers (six males and four females, mean age: 78.8 years) were examined to determine the distribution of the occipital cutaneous nerves after a detailed dissection. After inspecting the distribution patterns of the cutaneous nerves, the detailed relationship between the GON and the occipital artery (OA) was examined at the level of the SNL, which proceeded horizontally through the external occipital protuberance.

To visualize the innervation patterns, eight specimens were chosen from the four cadavers (three males and one female, mean age: 80.7 years). The specimen for observation was designated as the area where an incision line extended medially to a midsagittal line, laterally to a retroauricular line, superiorly to a vertex, and inferiorly to a trapezius attachment point including the SNL. Deep incisions to the bone were made to harvest the specimen after identifying the GON, LON, and TON via detailed dissection. The sections were separated from the bodies and were stained using the modified Sihler's method to visualize their innervation patterns.

Sihler's staining method rendered the muscle and connective tissue of the specimens transparent while the nerves remained visible. Details of the staining protocol were described in our previous studies. ${ }^{10,11}$ Briefly, unneutralized formalin (10\%) was inserted into the muscles via blood vessels. The muscle specimen was deposited into a $3 \%$ aqueous solution of potassium hydroxide with $3 \%$ hydrogen peroxide for 4 weeks. The specimen was decalcified using Sihler's solution I (1:1:6 of glacial acetic acid, glycerin, and 1\% aqueous chloral hydrate), and then stained with Sihler's solution II (1:1:6 of Ehrich's hematoxylin, glycerin, and 1\% aqueous chloral hydrate) for 4 weeks. Following this, the specimen was re-treated with Sihler's solution I for 30 minutes. The specimen was rinsed under tap water for 30 minutes and subsequently placed into a $0.05 \%$ lithium carbonate solution for 1 hour. Finally, the specimen was washed in increasing concentrations of glycerin (from $40 \%$ to $100 \%$ ).

Photographs of the stained specimen were taken with an illuminating plate, and the innervation pattern was manually traced.

\section{Analysis of the nerve distribution patterns of the specimens}

The specimens that had undergone Sihler's staining protocol were flattened (two dimensions), whereas the original specimens had three-dimensional shapes. Therefore, to interpret and compare the innervation patterns between the flattened and original specimens, topographically consistent landmarks had to be defined. For analysis of the distribution, the following landmarks were established (Figure 1): 

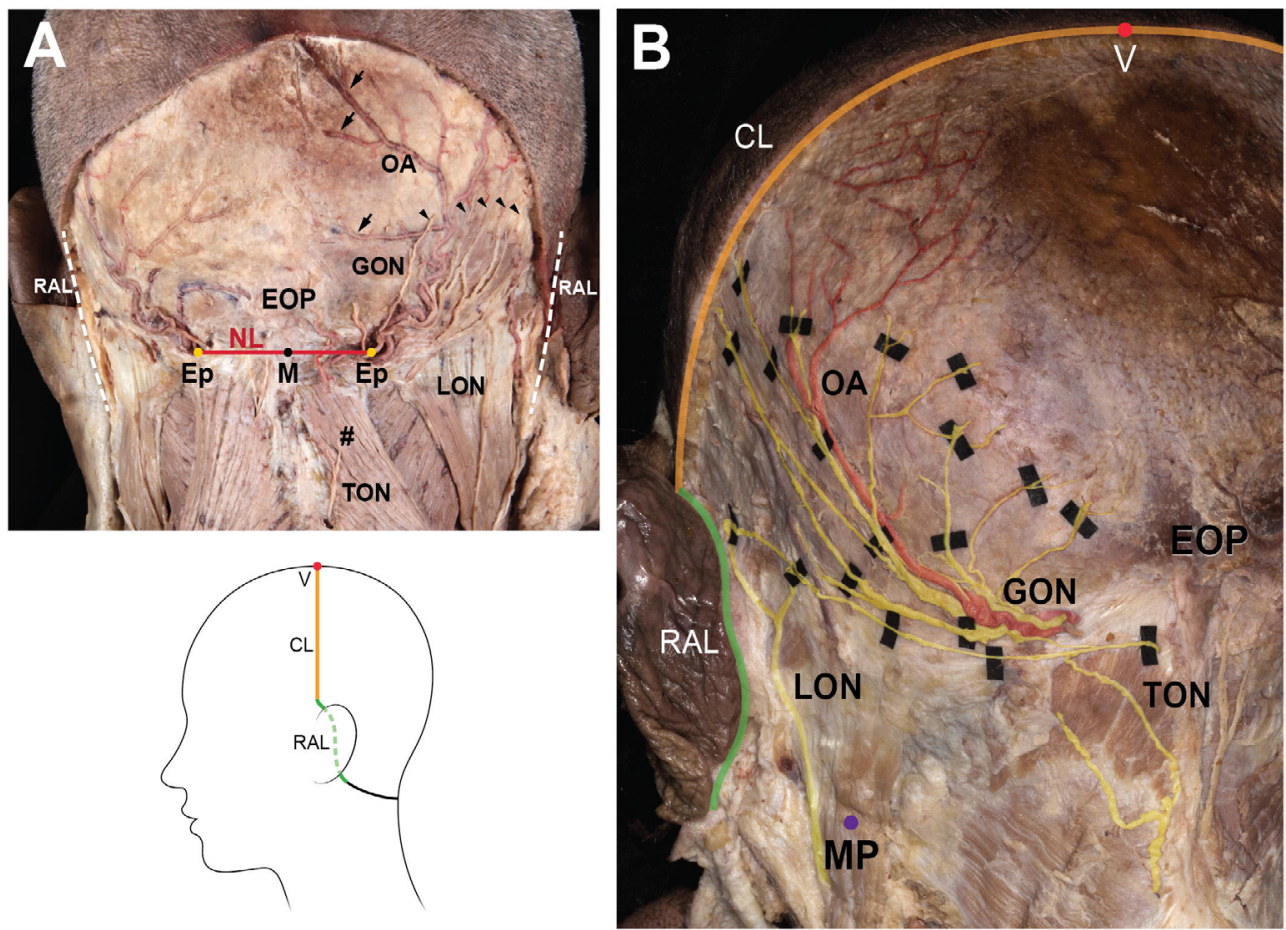

Figure I The landmark points and lines for specimen harvest (A) and the traced nerves and artery (B).

Notes: Arrowheads indicate the GON branches ascending laterally, and arrows indicate the OA branches heading toward the midline. The illustration in the lower column depicts the incision line that was made to harvest a specimen for Sihler's staining; \#, trapezius.

Abbreviations: $\mathrm{CL}$, coronal line through the $\mathrm{V}$ point; Ep, emerging point of GON; EOP, external occipital protuberance; GON, greater occipital nerve; M, midpoint of bilateral E points; MP, the lowermost point of mastoid process; NL, horizontal line through the bilateral E points; LON, lesser occipital nerve; OA, occipital artery; RAL, retroauricular line; TON, third occipital nerve; $\mathrm{V}$, vertex point.

1. V point: the uppermost point of the specimen, corresponding to the vertex

2. Ep: the area where the GON pierced the specimen

3. M point: midpoint of the bilateral Ep

4. Nuchal line (NL): the horizontal line through the bilateral Ep

5. Retroauricular line (RAL): the most lateral line of the specimen

6. Coronal line (CL): the quadrant circle of the superolateral margin of the specimen from the $\mathrm{V}$ point to the RAL.

Additionally, to understand the original topography of the specimen, it should be noted that an intensively stained area close to the NL nearly corresponded to the attachment point of the trapezius muscle and mastoid process or to the insertion of the sternocleidomastoid (SCM) muscle located between the Ep and the lowermost point of the RAL.

\section{Results}

\section{Inspection of the unstained specimens}

Dissected specimens without staining showed typical distributions of the GON, LON, TON, and OA (Figure 1B). They ascended laterally after piercing through rough connective tissue near the attachment point of the trapezius. The GON ascended and ramified into the occipital region, while the $\mathrm{OA}$ proceeded upwardly along the branches of the GON as one or two distinct vessels. Some branches of the OA reached the upper portion of the occipital region or midline. The LON ascended along the SCM and reached the retroauricular region. The TON appeared as short vertical twigs ascending medially to the GON, but usually ended before distributing into the area around the NL.

The detailed relationship between the GON and OA was examined at SNL region in the unstained specimens. In 12 of the specimens $(60 \%)$, the OA branches were located between the most medial and the most lateral branches (GONm and GONl, respectively) of the GON (Figure 1B). In four of the specimens $(20 \%)$, although the OA branches were located between the GONm and GONl, the most medial branch of the OA was located medial to the GONm in three specimens $(15 \%)$, while the most lateral branch of the OA was located lateral to the GON1 in one specimen $(5 \%)$. In three of the specimens (15\%), all of the OA branches were situated medial to the GON1. In the one remaining specimen (5\%), all of the OA branches were positioned lateral to the GON1. Regardless of the position of the OA relative to the GON, the arterial 
branches of the OA intermingled with the GON twigs as they ascended. Visible terminal branches of the OA tended to be directed more medially than the GON.

\section{Observations in specimens prepared with the modified Sihler's staining technique}

The distribution patterns of the GON and OA appeared very typical in the stained specimens. The GON was darkly stained after Sihler's staining and the OA appeared as vacant vestiges (Figure 2). We observed that the GON entered the specimen via the Ep, which was widened. Vicinal tissue around the NL and between the bilateral Ep was very tough and intensively stained. In all sections, the distinct branches of the GON proceeded laterally and extended from the superolateral area to the Ep. On the other hand, the GON was only thinly distributed in the middle area between the bilateral Ep. In particular, we did not observe any visible twigs in the middle-trisected area, which had a fan-like shape from the $M$ point to the CL. In six sections (75\%), the terminal branches of the GON reached the CL. In the two remaining sections (25\%), the terminal branches of the GON reached to the superior two-thirds of the line through the Ep to the CL. The terminal branches ended by turning medially toward the middle area.

In four of the sections (50\%), the GON ramified into the GONm and GONl after the Ep. They were easily detached, and thus we were able to count the number of nerve fibers in each group. The twigs of the GONm ascended vertically, with largely tortuous courses; these twigs were also more intensively congregated and exhibited more fibers when compared with the GONl (number of fibers: $5.6 \pm 1.3$ vs $2.4 \pm 1.1)$. Most of the twigs in the GONm did not reach the CL, as they turned medially. Additionally, twigs in the GONm were accompanied by OA branches. However, the OA branches extended to the vertex and middle areas of the occipital region. In contrast, the twigs of the GONl ascended obliquely, and diverged in a fan-like shape. Each twig in the GONl proceeded along a relatively straight course. In most of the sections, several terminal branches of the GONl reached the CL and RAL.

The LON was distinctly observed in the lateral half of the specimens, which was originally the mastoid region where the SCM was attached. Interestingly, twigs of the LON could be classified into the superficial and deep groups (Figure 3A).
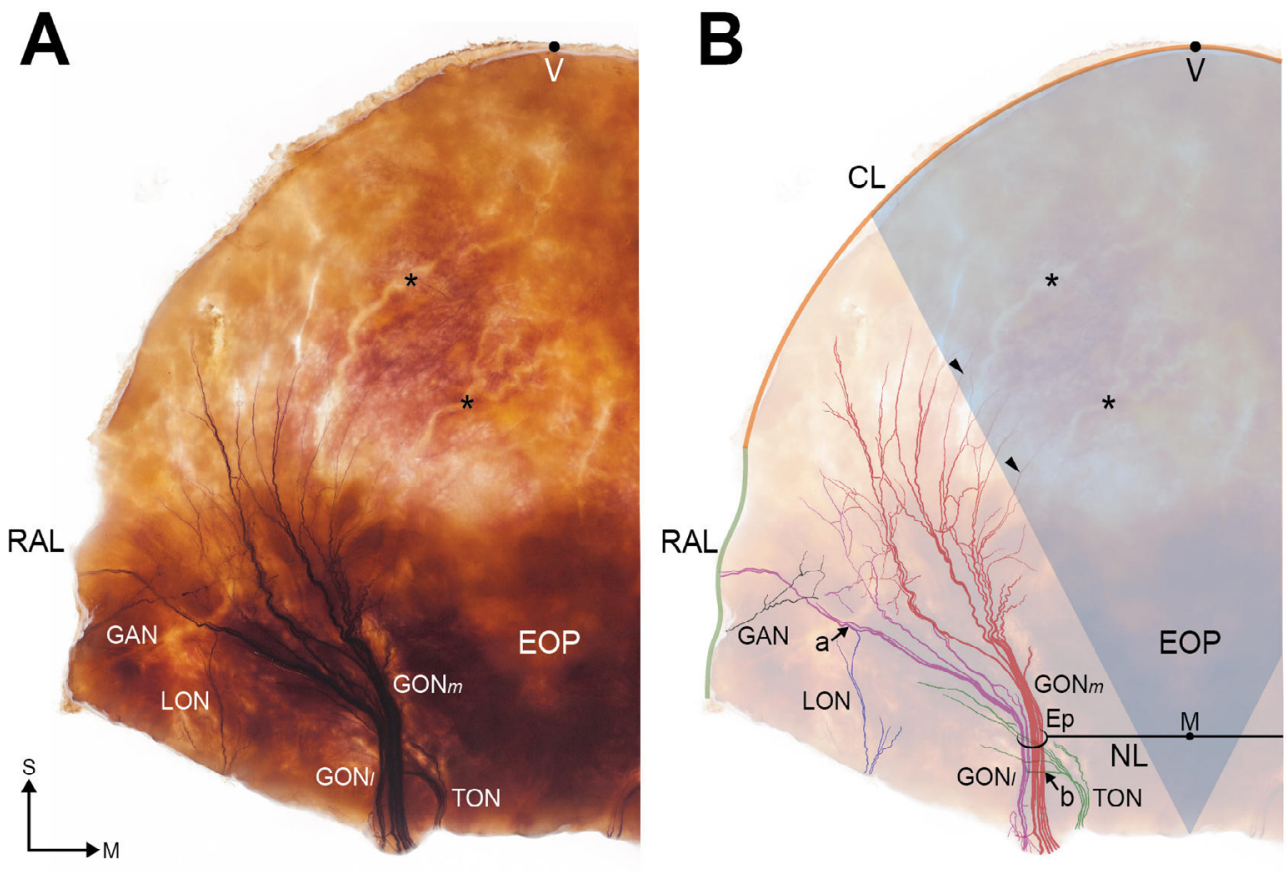

Figure 2 Overall distribution of the occipital cutaneous nerves in a specimen stained using the modified Sihler's staining protocol: (A) photograph of a typical specimen and (B) illustration showing the traced nerves.

Notes: Arrowheads indicate the terminal branches of the GONm, and asterisks (*) indicate the vacant vestiges of the OA branches. Arrow (a) indicates an area where the GONI and LON overlap, and arrow (b) indicates an area of overlap between the GON and TON. The bluish fan-like sector in B presents the nerve rarified area. $S$ and $M$ indicate the superior and medial directions.

Abbreviations: CL, coronal line through $\vee$ point; Ep, emerging point of GON; GON, greater occipital nerve; EOP, external occipital protuberance; GAN, great auricular nerve; GONm, medial group of GON; GONI, lateral group of GON; M, midpoint of bilateral E points; NL, horizontal line through the bilateral E points; LON, lesser occipital nerve; OA, occipital artery; RAL, retroauricular line; TON, third occipital nerve; $V$, vertex point. 
A layer of the LON deep group was mostly consistent with the GONl. Moreover, the deep group had twigs that anastomosed with the GON twigs. In six sections (75\%), the LON branches anastomosed or overlapped with the twigs of the GONl in the lateral half of the section near the RAL. We also observed LON twigs overlapping with the GON in the unstained specimens (Figure 3B).

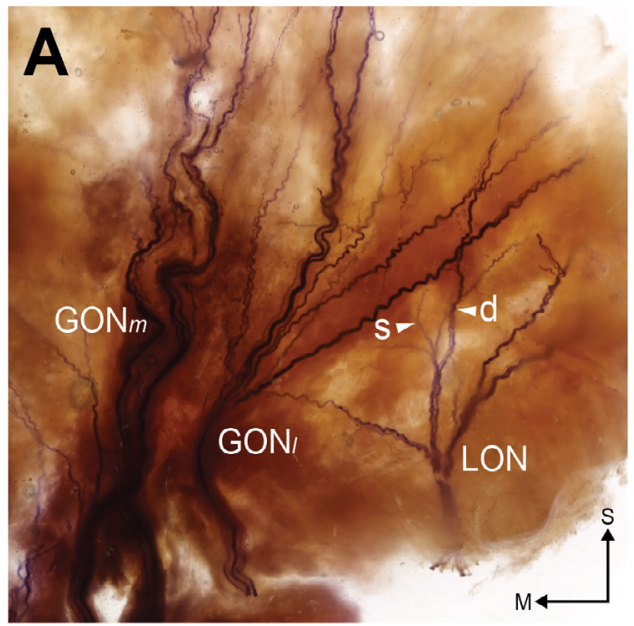

As for the TON, it was located medial to the Ep and was mostly examined outside. The TON did not vertically escape from the level of the NL region in any of the sections we evaluated (Figures 2 and 4). Some twigs of the TON joined with the GONl and some LON termini overlapped with the LON (Figure 4A and B). Additionally, we observed that horizontal branches of the TON traversed the GON and reached

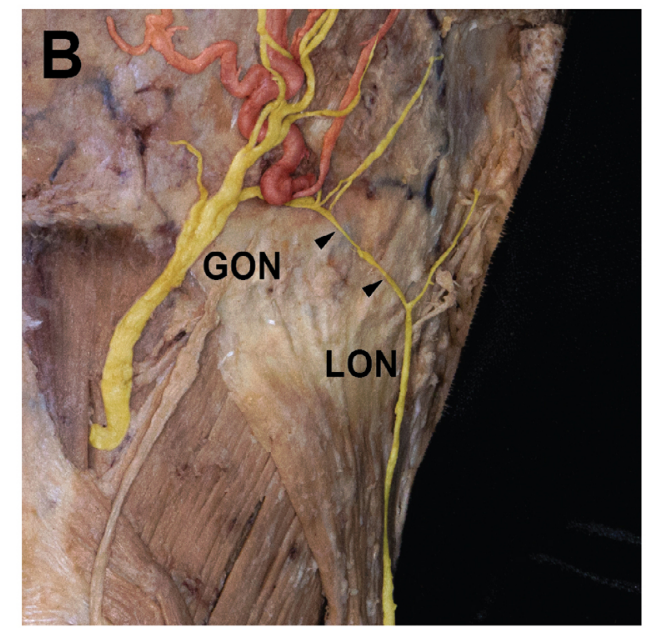

Figure 3 Anastomoses between the GON and LON in a stained specimen (A) and unstained specimen (B).

Notes: Arrowheads in A indicate superficial (s) and deep (d) twigs of the LON, and arrowheads in B indicate a distinct connecting branch between the GON and LON. S and $M$ indicate the superior and medial directions.

Abbreviations: GON, greater occipital nerve; GONm, medial group of GON; GONI, lateral group of GON; LON, lesser occipital nerve.
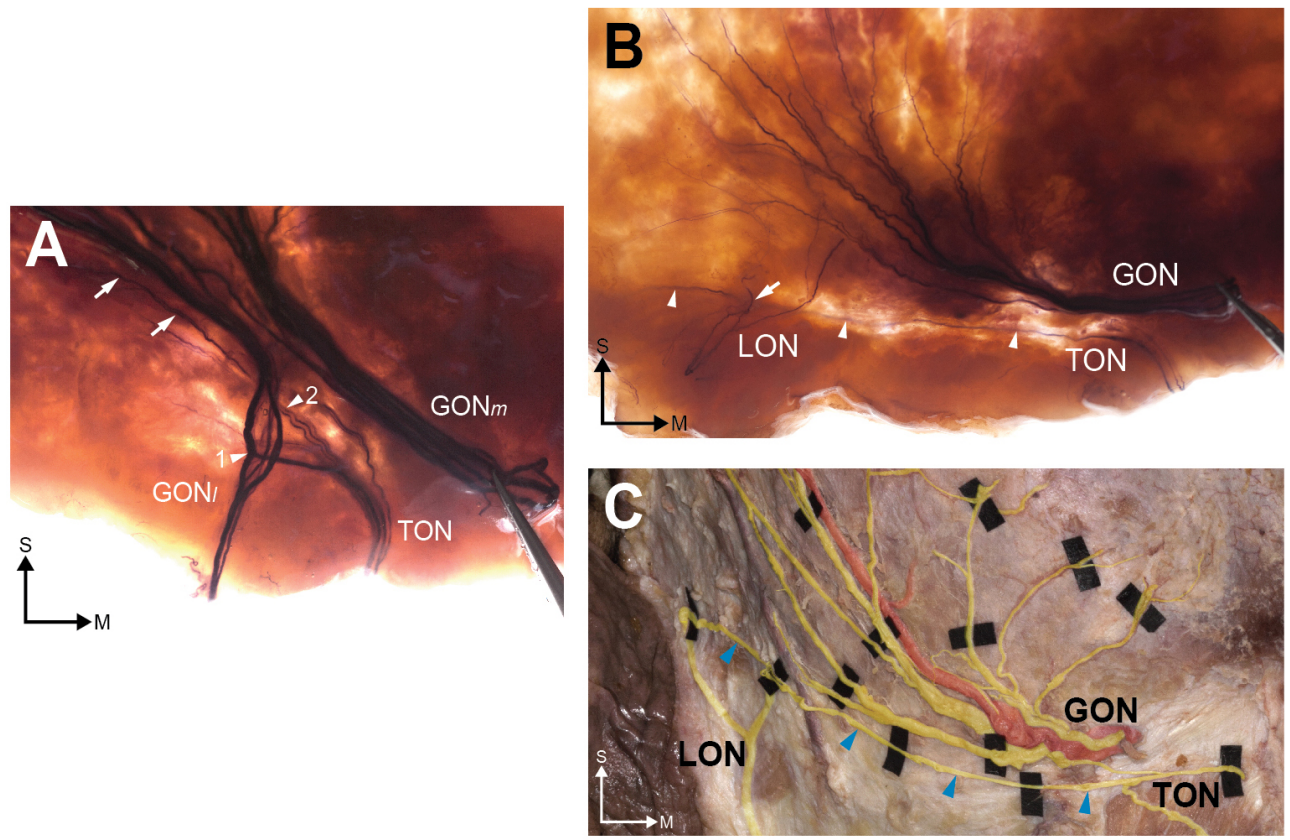

Figure 4 The TON and its relationship with the greater occipital nerve GON.

Notes: In the stained specimen (A), TON twigs cross (arrowhead I) or overlap (arrowhead 2) the fibers of the GON. A distinct horizontal twig (arrowheads) was observed in both the stained (B) and unstained (C) specimens. In (B), a twig of the lesser occipital nerve (LON; arrow) overlaps with the TON. S and M indicate the superior and medial directions.

Abbreviations: GON, greater occipital nerve; GONm, medial group of GON; GONI, lateral group of GON; LON, lesser occipital nerve; TON, third occipital nerve. 
the RAL in two of the stained specimens (Figure 4B) and in the unstained specimen (Figure 4C).

In all of the stained specimens, our visual evaluations revealed that the innervation patterns of the GON, LON, and TON were superimposed (Figure 5A); the typical distributions of these nerves are depicted in Figure 5B. In addition, the median angle between the midline and main branch of the GON was $22^{\circ}$ (range: $15-30^{\circ}$ ) (Figure $5 \mathrm{~B}$ ).

\section{Discussion}

Occipital neuralgia is defined as a painful syndrome with paroxysmal stabbing pain in the occipital region by the International Headache Society. ${ }^{5}$ One of the most common causes is an entrapment or compression of the occipital cutaneous nerves, the GON, LON, and TON. ${ }^{3}$ To alleviate the pain or to determine the origin of the nerve, an anesthetic block targeting the cutaneous nerves has been implemented. Thus, many studies have focused on the emergence of the cutaneous nerves. ${ }^{12-16}$ The GON has been known to have higher frequency (about 90\%) of engagement in the pain symptom. ${ }^{3}$ It serially passes or pierces the layered back muscles such as the obliquus capitis inferior (on $2.5-3 \mathrm{~cm}$ lateral to midline), the semispinalis capitis (on $1-1.5 \mathrm{~cm}$ inferior to the SNL and $3 \mathrm{~cm}$ lateral to midline), and the trapezius (on $1-1.5 \mathrm{~cm}$ inferior to the SNL and $2.5-3.5 \mathrm{~cm}$ lateral to midline). ${ }^{1,16,17}$ In the past, previous studies have focused on the emergence of the nerves on the layered back muscles for guiding nerve blocks. However, the detailed distribution of the cutaneous nerve fibers should be examined for diagnosis and treatment of the nerve triggering the pain. Up to now, the fine distribution patterns of the nerves have been veiled since it is very difficult to determine the distribution of their tiny termini by manual dissection.

The present study used the modified Sihler's staining method to visualize the courses and distribution patterns of the GON, LON, and TON in the occipital region. The major finding of this study was that the distribution of the GON was mostly concentrated toward the superolateral area in the occipital region. The distribution areas of the LON and TON were biased toward the lateral side and below the SNL, but these nerves exhibited multiple interconnections or areas of overlap with the GON. Furthermore, we observed a nerve rarified zone in the shape of an inverted triangle in the middle occipital area.

Here, we found that the GON-rich zone was mostly concentrated in the superolateral area of the posterior scalp. Interestingly, the stained specimens typically did not show long vertical GON twigs directly reaching the middle upper area of the occipital region, including the vertex, in this study. Physiologically, this middle upper area has somatic sensation, and thus tiny GON twigs, which could not be observed in the stained specimens in the present study, innervate this
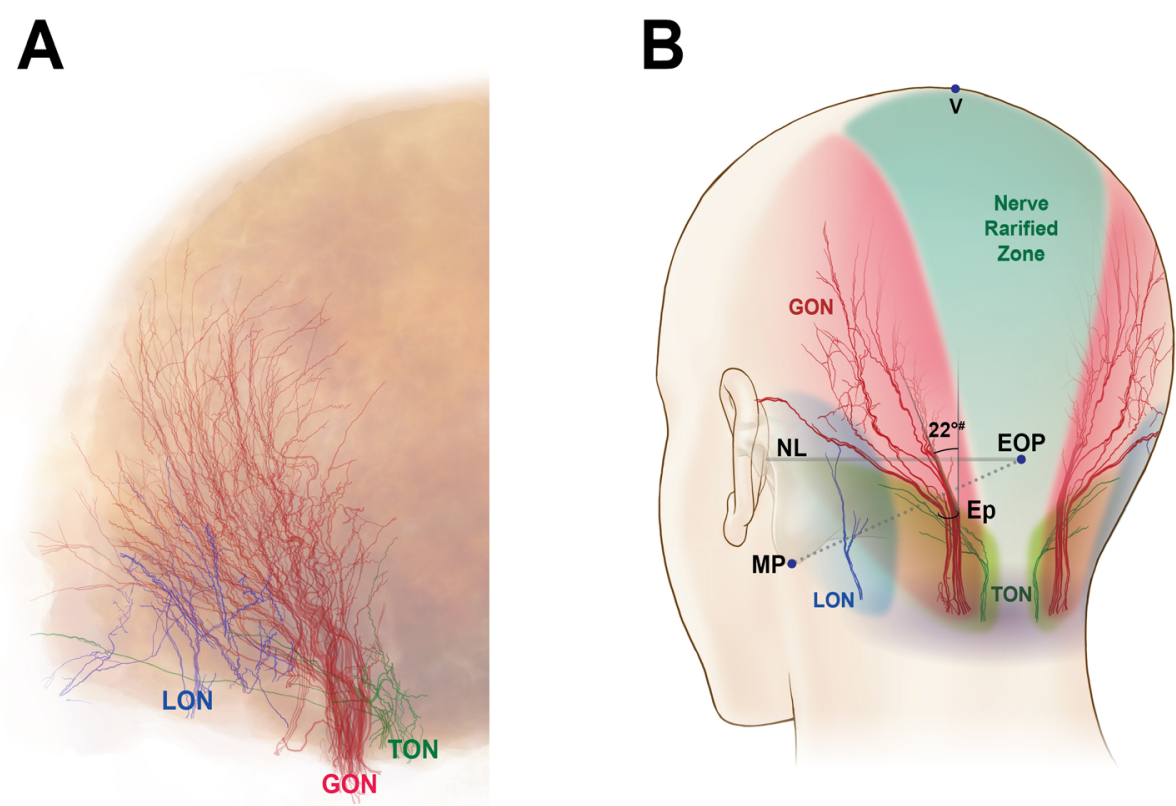

Figure 5 Overall innervation of the occipital cutaneous nerves.

Notes: The traced nerves in all specimens were superimposed $(\mathbf{A})$ and a typical innervation distribution pattern is shown (B). A nerve rarified zone is visible in the middle of the trisected area (green). The median angle (\#) between the most medial observed twig of the GON and a vertical line is $22^{\circ}$.

Abbreviations: Ep, emerging point of GON; EOP, external occipital protuberance; GON, greater occipital nerve; LON, lesser occipital nerve; MP, the lowermost point of mastoid process; NL, horizontal line through the bilateral $E$ points; TON, third occipital nerve; $V$, vertex point. 
region. Our study revealed that the GONm was a large trunk, with a greater number of fibers than the GONl. Therefore, we speculate that the medial fibers of the GON are distributed throughout the middle upper area of the occipital region. Clinically, our study implies that pain triggered by the GON itself is more dominant on the superolateral side than in the midline area of the occipital region, although various referred pain patterns originating from the upper cervical segment may exist in the head. ${ }^{4,12}$ Specifically, based on our results, entrapment neuropathies, such as the typical occipital neuralgia that occurs following traumatic or surgical events, may present as radiating pain to the superolateral side rather than as pain in the middle upper area of the occipital region.

The present findings may suggest that, when a GON block is performed, superolateral spreading of the anesthetic agent from the entry point might be preferable over spreading in only the superior direction to the vertex or medial side. To confirm this assumption, we compared the injectate spread patterns between the superolateral $\left(\sim 20-25^{\circ}\right.$ to the sagittal line) and vertical needle tip directions at the recommended injection point $(2 \mathrm{~cm}$ lateral and $2 \mathrm{~cm}$ inferior to the external occipital protuberance $)^{13}$ in the fresh cadaver of an 81-yearold man (Figure 6). When $2 \mathrm{~mL}$ of dye was mixed with the injectate, the superolaterally injected side displayed a more favorable spread pattern, encompassing all of the GON branches. However, when the GON block was performed using the vertical injection technique, we observed faint or no staining in the GON1. A local injection into the emerging point of the GON in the upper trapezius or $\mathrm{C} 2$ level over the oblique capitis inferior muscle, before bifurcation of the GON into the GONm and GON1, produced sufficient anesthesia of the entire GON. ${ }^{6,7,13}$ However, blind identification of this optimal point might be difficult. Moreover, the GONm and

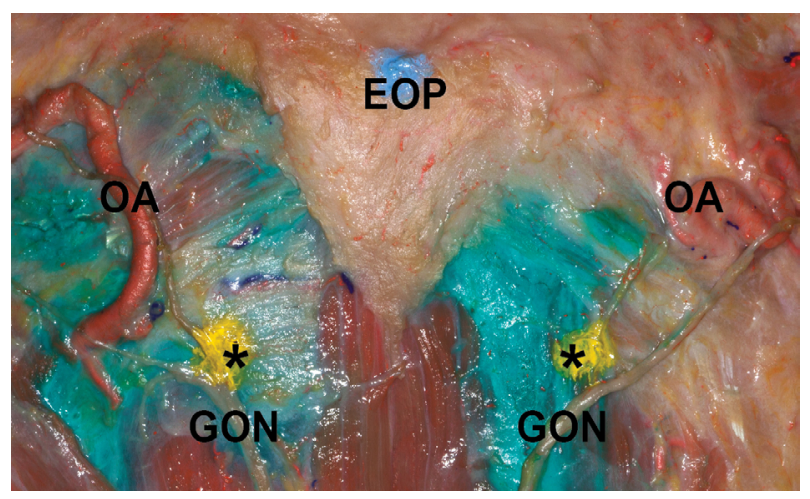

Figure 6 Comparison of the dye (green) spread patterns between superolateral (left) and vertical (right) injections at the recommended point $\left(^{*}\right)$ for GON blocks. ${ }^{13}$ Abbreviations: EOP, external occipital protuberance; GON, greater occipital nerve; OA, occipital artery.
GONl already existed at the SNL level, which is a commonly used site when employing the OA palpation method for GON blocks. ${ }^{3,6,14}$ Thus, performing a GON block with the needle in the superolateral direction may produce proper spreading of the injectate. It should also be noted that although most of the OA branches we observed in this study were between the GONm and GONl at the SLN, with courses that were mainly parallel to the GON, typical locations of the OA branches could not be established in this study due to their high variability. Nevertheless, because multiple needle punctures, such as those made in a fan-like manner, for GON blocks could result in potential tissue or vessel injury and require larger volumes of local anesthetics, understanding the distribution patterns of the GON is essential for ensuring that a proper needle technique is used. Thus, our findings regarding the distribution of the GON will aid clinicians in generating successful and safe GON blocks.

Although the GON is the main target of nerve blocks and other neuromodulations for controlling occipital pain, the LON and TON have also been studied as potential sources of occipital pain conditions such as occipital neuralgia or cervicogenic headache. ${ }^{3,4,12,15}$ Our evaluations showed that the LON did not innervate the main occipital region and had limited lower lateral and retroauricular distributions in all examined sections. According to our previous study, where the reference point is external occipital protuberance, TON pierced the SSC at $1.2 \pm 1 \mathrm{~cm}$ lateral and $6.1 \pm 1.5 \mathrm{~cm}$ inferior, the splenius capitis at $1 \pm 0.5 \mathrm{~cm}$ lateral and $5.9 \pm 2 \mathrm{~cm}$ inferior, and the trapezius at $1.3 \pm 0.9 \mathrm{~cm}$ lateral and $4.4 \pm 2.1 \mathrm{~cm}$ inferior. ${ }^{17}$ It is well known that this nerve is typically located medial to the GON at the cervico-occipital level. ${ }^{16,17}$ However, we found that the TON did not appear to extend above the SNL. No stained TON branches were observed medial to the GON above the SLN to the vertex in this study. Despite the limited distributions of the LON and TON in the lower occipital region, our study using Sihler's staining confirmed the presence of multiple interconnections or areas of overlap among the LON, TON, and GON that had been reported previously by several gross anatomical studies. ${ }^{1,3,18}$ Importantly, the present study clearly shows that some branches of the TON traverse horizontally and cross the GONl, whereas previous descriptions only noted that the branches ascend vertically. ${ }^{19}$ Collectively, our anatomical findings explain the complex pain patterns that are often experienced in the occipital regions. Moreover, our results imply that it is likely very difficult to achieve selective or targeted nerve blocks for each occipital nerve above the cervical level in a clinical setting. 
In the present study, we utilized both preparations and described the anatomy in each. To do this, we defined reference landmarks within the occipital region. However, herein, we only provided qualitative, not quantitative, descriptions of the topography of the cutaneous nerves. Further studies utilizing quantitative measurements may provide additional useful information regarding the distribution patterns of the nerves. For instance, in the middle area, although we did not observe any nerve twigs, this does not necessarily mean that sensory twigs were absent in this area, but rather that they are rare. An immunohistologic examination using antibodies for the axons, such as NF200, may provide information on the axon intensity. In addition, since our findings from the GON blocks we performed with different needle directions suggest that the needle trajectory for GON blocks may affect the injectate spread pattern, a clinical study to determine the practical significance of these results is warranted.

\section{Conclusion}

This anatomical study using Sihler's staining yielded new information regarding the detailed distributions of major occipital nerves. Specifically, we found that the branches of the GON are mostly concentrated toward the superolateral area from its emerging point. The distribution areas of the LON and TON were small and limited, but we identified multiple interconnections and areas of overlap among these nerves and the GON. We also observed a nerve rarified zone of the occipital nerves in the middle occipital area. Collectively, our findings improve our understanding of the anatomy of the occipital nerves, which may aid in the management of occipital pain in clinical practice.

\section{Acknowledgments}

This work was supported by the National Research Foundation of Korea (NRF) grant funded by the Korea government (MSIT) (NRF-2017R1C1B5074007). This cadaveric anatomical study was approved by the institutional review board of Severance Hospital, Yonsei University Health System (No 4-2017-1180). All cadavers utilized in the present study were legally donated to the Surgical Anatomy Education Center at Yonsei University College of Medicine. The authors thank the noble contributors who donated their bodies to the institutions. The present research would not be possible without their selfless and precious gift. We are grateful to Dae Won Kim, Jun Ho Kim, and Jong Ho Bang for their technical support (all are staff members in the Surgical Anatomy Education Center at the Yonsei University College of Medicine).

\section{Disclosure}

The authors report no conflicts of interest in this work.

\section{References}

1. Shin KJ, Kim HS, O J, Kwon HJ, Yang HM. Anatomical consideration of the occipital cutaneous nerves and artery for the safe treatment of occipital neuralgia. Clin Anat. Epub 2018 May 12.

2. Kim H-S, Shin K-J, O J, et al. Stereotactic topography of the greater and third occipital nerves and its clinical implication. Sci Rep. 2018;8(1):870.

3. Choi I, Jeon SR. Neuralgias of the Head: Occipital Neuralgia. J Korean Med Sci. 2016;31(4):479-488.

4. Gadient PM, Smith JH. The neuralgias: diagnosis and management. Curr Neurol Neurosci Rep. 2014;14(7):459.

5. Headache Classification Committee of the International Headache Society (IHS). The International Classification of Headache Disorders, 3rd edition (beta version). Cephalalgia. 2013;33(9):629-808.

6. Greher M, Moriggl B, Curatolo M, Kirchmair L, Eichenberger U. Sonographic visualization and ultrasound-guided blockade of the greater occipital nerve: a comparison of two selective techniques confirmed by anatomical dissection. Br J Anaesth. 2010;104(5): 637-642.

7. Pingree MJ, Sole JS, O' Brien TG, Eldrige JS, Moeschler SM. Clinical efficacy of an ultrasound-guided greater occipital nerve block at the level of C2. Reg Anesth Pain Med. 2017;42(1):99-104.

8. Won SY, Kim DH, Yang HM, et al. Clinical and anatomical approach using Sihler's staining technique (whole mount nerve stain). Anat Cell Biol. 2011;44(1):1-7.

9. Liu AT, Yu DZ, Chen G, et al. Profiling of innervations of mimetic muscles in fresh human cadavers using a modified Sihler's technique. Muscle Nerve. 2010;42(1):88-94.

10. Yang HM, Won SY, Lee YI, Kim HJ, Hu KS. The Sihler staining study of the infraorbital nerve and its clinical complication. J Craniofac Surg. 2014;25(6):1-2213.

11. Yang HM, Won SY, Kim HJ, Hu KS. Sihler staining study of anastomosis between the facial and trigeminal nerves in the ocular area and its clinical implications. Muscle Nerve. 2013;48(4):545-550.

12. Bogduk N. Cervicogenic headache: anatomic basis and pathophysiologic mechanisms. Curr Pain Headache Rep. 2001;5(4):382-386.

13. Loukas M, El-Sedfy A, Tubbs RS, et al. Identification of greater occipital nerve landmarks for the treatment of occipital neuralgia. Folia Morphol. 2006;65(4):337-342.

14. Lauretti GR, Corrêa SW, Mattos AL. Efficacy of the greater occipital nerve block for cervicogenic headache: Comparing classical and subcompartmental techniques. Pain Pract. 2015;15(7): 654-661.

15. Jung SJ, Moon SK, Kim TY, Eom KS. A case of occipital neuralgia in the greater and lesser occipital nerves treated with neurectomy by using transcranial Doppler sonography: technical aspects. Korean $J$ Pain. 2011;24(1):48-52.

16. Kariya K, Usui Y, Higashi N, et al. Anatomical basis for simultaneous block of greater and third occipital nerves, with an ultrasound-guided technique. J Anesth. 2018;32(4):483-492.

17. Kim HS, Shin KJ, O J, et al. Stereotactic topography of the greater and third occipital nerves and its clinical implication. Sci Rep. 2018;8(1):870.

18. Cesmebasi A, Muhleman MA, Hulsberg P, et al. Occipital neuralgia: anatomic considerations. Clin Anat. 2015;28(1):101-108.

19. Tubbs RS, Mortazavi MM, Loukas M, et al. Anatomical study of the third occipital nerve and its potential role in occipital headache/neck pain following midline dissections of the craniocervical junction. J Neurosurg Spine. 2011;15(1):71-75. 
The Journal of Pain Research is an international, peer reviewed, open access, online journal that welcomes laboratory and clinical findings in the fields of pain research and the prevention and management of pain. Original research, reviews, symposium reports, hypothesis formation and commentaries are all considered for publication
The manuscript management system is completely online and includes a very quick and fair peer-review system, which is all easy to use. Visit http://www.dovepress.com/testimonials.php to read real quotes from published authors.

Submit your manuscript here: https://www.dovepress.com/journal-of-pain-research-journal 